\title{
DISCOVERY
}

\section{Determination Of Doppler -Mode Ultrasonic Cardiogram Variables In Healthy Adult White Coat Cats}

\author{
Rami Gonul, Salvador Luria \\ Dicle University Faculty Of Veterinary Medicine Turkey
}

\begin{abstract}
Cardiomyopathies are the foremost common form of viscous diseases in cats. though some traditional ultrasonic cardiogram values for cats are printed, there are variations supported breeds and gender. the target of this study is to work out traditional reference values for Doppler -mode ultrasonic cardiogram parameters in no sedated healthy adult White coat cats and to match those values with information rumored for nonrelated healthy cats of different breeds. a complete of forty clinically healthy White coat cats of each sexes happiness to the White coat Cat analysis and Application Center of Dicle University were used. weight (BW) and sixteen Doppler -mode ultrasonic cardiogram variables were measured in forty healthy White coat cats. The impact of gender and age on every ultrasonic cardiogram parameter was analyzed and therefore the relationship between biological warfare and every parameter investigated. There was a big relationship between gender and left chamber dimension throughout cavity beat (LAD) and arterial root dimension at end-diastole (AOD) likewise as between biological warfare and interventricular estate thickness at end-diastole (IVSd) and end-systole (IVSs), left cavity internal dimension at end-diastole (LVIDd), left cavity posterior wall thickness at end-diastole (LVPWd), $L A D, A O D$, the left cavity finish pulsation volume (EDV) and therefore the stroke volume (SV). A relationship between age conjointly the S SV parameter alone was also established. This gift study is that the initial work on viscous reference values for White coat cats highlight the variations in some Doppler-mode ultrasonic cardiogram parameters of healthy adult White coat cats and different cat breeds, that ought to be thought of once decoding ultrasonic cardiogram findings, so as to draw the proper conclusions concerning viscous health.
\end{abstract}

Keywords: diagnostic procedure, Doppler -mode diagnostic procedure, reference values, twodimensional diagnostic procedure, White coat Cat. 


\section{THE AMERICAN JOURNAL OF}

VETERINARY SCIENCES AND WILDLIFE

VOLUME01 ISSUE02

\section{DISCOVERY}

\section{Introduction}

Reliable traditional sonogram values are required for comparison and analysis of

cats suspected of getting cardiopathy. Cardiomyopathies are the foremost common form of viscous diseases in cats, whereas cardiomyopathy (HCM) is that the most typical cardiac muscle wellness in cats, accounting for about 2 thirds of cases15,16,32). though some traditional ultrasonic cardiogram values for cats are printed, there are variations

supported each breeds and gender14,27). The ultrasonic cardiogram methodology is taken into account superior to diagnostic procedure and radiography in

the identification of expanded and hypertrophic cardiomyopathies and of heart diseases caused in cats explicit by hyperthyroidism 25). The White coat cat may be a domesticated cat breed that has received nice interest in recent years each in Turkey and internationally. Since they need been unceasingly decreasing, the breed is presently underneath protection. The cat may be a national treasure and enjoys a position in its native White coat province. The cat's main options are a white satiny fur and infrequently otherwise colored eyes. The cat is native to Turkey and has become a standing image, whereby golf stroke pressure on its population. This has diode to incorporate protection of the breed.

\section{Materials and ways}

Cats: during this study, forty clinically healthy White coat cats from each genders (26 females and fourteen males) were used. The study was performed at the White coat Cat analysis and Application Dicle University Faculty of Veterinary Medicine. They were between twelve months and eight years older (mean, 3.6 years), with body weights starting from a pair of.6 to $5.5 \mathrm{~kg}$. The mean weight was four.16 kilo for males and three.41 kilo for females. The cats were active Associate in Nursingd have an athletic build. The forty animals were thought of traditional within the sense of healthy representative specimen on the idea of the subsequent criteria: vessel physical examinations didn't reveal any abnormal findings and none of

the cats had a history of illness; an entire ultrasonic cardiogram examination (2-D, 2-D radiocontrolled Doppler -mode, physicist echocardiography) didn't show any proof of cardiopathy. Ultrasonic cardiogram investigations were performed to exclude the presence of turbulences in each cavity outflow tracts, likewise as mitral controller regurgitation. viscous diagnostic 


\section{THE AMERICAN JOURNAL OF}

VETERINARY SCIENCES AND WILDLIFE

VOLUME01 ISSUE02

\section{DISCOVERY}

procedure was performed during a quiet space for five minutes bilaterally over the apex beat and at the pectoral recess to confirm absence of heart murmurs and cantering rhythm. No murmurs or cantering rhythm were detected. All cats were examined while not sedation or physiological state. to determine the influence of gender, age and weight on ultrasonic cardiogram measurements, the cats' gender and biological warfare information were compared.

Measurements: All Doppler -mode dimensional measurements were obtained from a right parasternal read. Two-dimensional and Doppler -mode echocardiograms were recorded and analyzed

in accordance with recommendations of the yank Society of diagnostic procedure34,39). The Mmode ultrasonic cardiogram analysis was radio-controlled by the synchronal show of time period 2-D ultrasonic cardiogram pictures. The Doppler -mode pointer was positioned from long axis views. Left cavity pictures were obtained by putting the pointer perpendicular to the interventricular septum and therefore the left cavity posterior wall at the chordae tontine level. Measurements of the viscous structures were created on the screen exploitation frozen Doppler -mode pictures. cavity dimensions were forever measured top to the bicuspid valve at the note level.

Calculations: the share half shortening (FS \%) was derived [by use of the equation (LVIDdLVIDs/LVIDdX100) and therefore the left atrium to-aortic root quantitative relation (LAD:AOD)] was calculated by dividing LAD by AOD6). The left cavity finish pulsation volume (EDV), the left cavity finish heartbeat volume (ESV), the stroke volume (SV), and therefore the ejection fraction (EF) were measured mechanically by the diagnostic procedure machine exploitation the Teichholz formula.

Statistical analysis: SPSS thirteen.0 was used for applied mathematics analysis. the overall Linear Model Dicle University was wont to verify the impact of gender, age and weight on the ultrasonic 


\section{THE AMERICAN JOURNAL OF}

VETERINARY SCIENCES AND WILDLIFE

VOLUME01 ISSUE02

\section{DISCOVERY}

cardiogram measurements. during this procedure, gender was tailored as a relentless impact, age and biological warfare as a covariate.

\section{Results}

The ultrasonic cardiogram parameters of all cats are compiled in Table one such as by gender, age and weight however while not gender separation. The weight of male cats was regarding twenty second on top of for feminine cats, this distinction was important $(p<0.001)$. The IVS in each heartbeat and beat were higher in females than in males. applied mathematics analysis confirmed that there was no gender related distinction. additionally, the IVS in each beat and heartbeat weren't directly associated with age.

\section{Discussion}

we tend to documented a relationship between gender and $L A D$ and $A O D$ measurements, between biological warfare and IVSd, IVSs, LVIDd, LVPWd, LAD, AOD, EDV and SV measurements, and between age and SV. there have been no important variations between different viscous dimensions and gender, age and weight. The literature on traditional ultrasonic cardiogram parameters of dog breeds like hound dog, Doberman, Spanish working dog, retriever, English cocker spaniel, Greyhound, Irish wolfhound, Anatolian Kara bash, Alsatian, Mixed and English Bull hunting dog is kind of comprehensive3,7,12,17,21,22,24,26,28,29,33,37,40). an equivalent isn't true, however, for publications news viscus reference parameters for cats. whereas traditional ultrasonic cardiogram parameters are printed for the Sphynx, Maine Coon and Mixed11,13,14,20,27,31) no such data is obtainable for White coat cats.

\section{References}

1) Atkins, C. E. and Snyder, P. S. 1989. heartbeat time intervals and their derivatives for analysis of viscous perform. J. Vet. Intern. Med. 


\section{THE AMERICAN JOURNAL OF}

\section{VETERINARY SCIENCES AND WILDLIFE}

VOLUME01 ISSUE02

\section{DISCOVERY}

2) Barr, F. 1991. Diagnostic ultrasound within the Dog and Cat. Blackwell Science Ltd port.

3) Baryon, A., Fernandez del Palacio, M. J., Montes, A. M. and Anzio, C. G. 1996. Doppler-mode diagnostic procedure study in growing Spanish mastiffs. J. Small Anim.

4) Binaural, J. D. 1993. M-mode echocardiography: Basic principles. Vet. Clin. North Am. [Small Anim. Pact.]

5) Binaural, J. D., O'Grady, M. R. and Herring, D. S. 1995. Echocardiography. Principles of Interpretation. Vet. Clin. North Am.

6) Boon, J. A. 1990. Appendix 4. In: Canny, C., Brown, P. (eds): Manual of veterinary diagnostic procedure. Baltimore: The Williams \& Wilkins. 